This is the post peer-review accepted manuscript of:

M. Tognon , A. Testa , E. Rossi and A. Franchi , "Takeoff and Landing on Slopes via Inclined Hovering with a Tethered Aerial Robot", 2016 International Conference on Intelligent Robots and Systems (IROS), Daejeon, Korea, 2016, pp. 1702-1707.

The published version is available online at:

https://doi.org/10.1109/IROS.2016.7759273

(C) 2019 IEEE. Personal use of this material is permitted. Permission from IEEE must be obtained for all other uses, in any current or future media, including reprinting /republishing this material for advertising or promotional purposes, creating new collective works, for resale or redistribution to servers or lists, or reuse of any copyrighted component of this work in other works. 


\title{
Takeoff and Landing on Slopes via Inclined Hovering with a Tethered Aerial Robot
}

\author{
Marco Tognon ${ }^{1}$, Andrea Testa ${ }^{2}$, Enrica Rossi ${ }^{1}$ and Antonio Franchi ${ }^{1}$
}

\begin{abstract}
In this paper we face the challenging problem of takeoff and landing on sloped surfaces for a VTOL aerial vehicle. We define the general conditions for a safe and robust maneuver and we analyze and compare two classes of methods to fulfill these conditions: free-flight vs. passivelytethered. Focusing on the less studied tethered method, we show its advantages w.r.t. the free-flight method thanks to the possibility of inclined hovering equilibria. We prove that the tether configuration and the inclination of the aerial vehicle w.r.t. the slope are flat outputs of the system and we design a hierarchical nonlinear controller based on this property. We then show how this controller can be used to land and takeoff in a robust way without the need of either a planner or a perfect tracking. The validity and applicability of the method in the real world is shown by experiments with a quadrotor that is able to perform a safe landing and takeoff on a sloped surface.
\end{abstract}

\section{INTRODUCTION}

The attention that aerial robotics is having over the last decade is constantly increasing. This is mainly due to the technological progress that makes the aerial vehicles adequate for a broad variety of applications, e.g., search and rescue, where the task consists on providing assistance in hostile environments such as mountains or civil areas after natural catastrophes. In this scenarios it is very likely that the terrain is not flat, making the landing and takeoff maneuvers very complicate and unsafe. The problem of landing on a sloped (not flat) surface is a very challenging problem for a VTOL (Vertical Take-Off and Landing) vehicle due to its underactuation. In fact the task requires to control both position and attitude since the vehicle has to be oriented as the surface, but this is not possible.

For this problem we can identify two solution categories: i) free-flight and ii) tethered methods. The classical approach for free-flying vehicles is based on motion planning [1][3] (sometimes called perching maneuver). It consists on exploiting the flatness of the system with respect to the position [4] to plan a desired trajectory such that the vehicle ends the maneuver with the proper position and orientation. Different controllers can be then applied to track this trajectory. The success of the maneuver requires an almost perfect tracking that implies an almost perfect knowledge of the model. Otherwise, small deviations from the nominal trajectory would lead to miss the target or to crash on it.

On the other hand, a method gaining more and more interest, consists on the use of a tether that links the vehicle to a

\footnotetext{
${ }^{1}$ LAAS-CNRS, Université de Toulouse, CNRS, Toulouse, France, mtognon@laas.fr, erossi@laas.fr, antonio.franchi@laas.fr

${ }^{2}$ Department of Engineering, Università del Salento, via per Monteroni, 73100 Lecce, Italy andrea.testa@unisalento.it

Partially funded by the European Union's Horizon 2020 research and innovation programme under grant agreement No 644271 AEROARMS.
}

fixed or moving point on the ground. Previous works already presented different controllers for the 2D environment [5], [6]. Furthermore, in works like [7]-[9] the tether, together with an actuated winch, were proposed to face the problem of landing and takeoff on flat moving surfaces. Using this solution one could simply pull the vehicle toward the ground, as done in [7], [8]. Nevertheless, this configuration requires to add an actuator that increases the complexity of the system and reduces its already limited payload if placed on-board. For these reasons we instead study the case of a passive tether that does not require extra actuation. For this system we analyze its intrinsic characteristics proving the differential flatness with respect to the outputs that are of interest for the landing and takeoff. Exploiting this property, we then design a controller based on hierarchical techniques.

One of the main contributions of this paper is the definition of some general conditions to perform a robust takeoff and landing. We then provide a careful analysis and a comparison of the free-flight and passive-tethered method, based on these conditions. This study shows that, when an anchoring spot is available, the tether solution is highly preferable with respect to the free-flight one since it is the only one that allows to land on any sloped surface, and with good repeatability and robustness to tracking inaccuracies. Furthermore, we also design a method to pass from the free-flight to the tether condition. The global method is finally tested through exhaustive real experiments in which a quadrotor is able to perform the landing and takeoff on/from a sloped surface.

The paper is organized as follows. In Sec. II we model the system. In Sec. III we define the conditions for the landing and takeoff maneuver. The analysis of the free-flight and tethered methods is done in Sec. IV. Then, in Sec. V the differential flatness is proven and we design a hierarchical controller. In Sec. VI the method is experimentally validated. Conclusions and future works are discussed in Sec. VII.

\section{Modeling}

We denote the world frame by $\mathscr{F}_{W}=O_{W},\left\{\mathbf{x}_{W}, \mathbf{y}_{W}, \mathbf{z}_{W}\right\}$. Given an aerial robot, we define a frame rigidly attached to it, $\mathscr{F}_{R}=O_{R},\left\{\mathbf{x}_{R}, \mathbf{y}_{R}, \mathbf{z}_{R}\right\}$, where $O_{R}$ is set on the vehicle center of mass $(\mathrm{CoM})$. The position of $O_{R}$ in $\mathscr{F}_{W}$ is described by the vector $\mathbf{p}_{R} \in \mathbb{R}^{3}$, while its orientation with respect to $\mathscr{F}_{W}$ is given by the rotation matrix $\mathbf{R}_{R} \in \mathrm{SO}(3)$. The angular velocity of $\mathscr{F}_{R}$ with respect to $\mathscr{F}_{W}$, expressed in $\mathscr{F}_{R}$, is denoted by $\boldsymbol{\omega}_{R} \in \mathbb{R}^{3}$. The aerial vehicle is a VTOL modeled as a rigid body with mass $m_{R} \in \mathbb{R}_{>0}$ and positive definite inertia matrix $\mathbf{J}_{R} \in \mathbb{R}^{3 \times 3}$ (expressed in $\mathscr{F}_{R}$ and relative to $O_{R}$ ). Its motion in the 3D space is controlled by four control inputs: $f_{R} \in \mathbb{R}$ and $\tau_{R}=\left[\begin{array}{lll}\tau_{R x} & \tau_{R y} & \tau_{R z}\end{array}\right]^{T} \in \mathbb{R}^{3}$, where $f_{R}$ is the magnitude of the thrust force $\mathbf{f}_{R}=-f_{R} \mathbf{z}_{R}$ applied at 


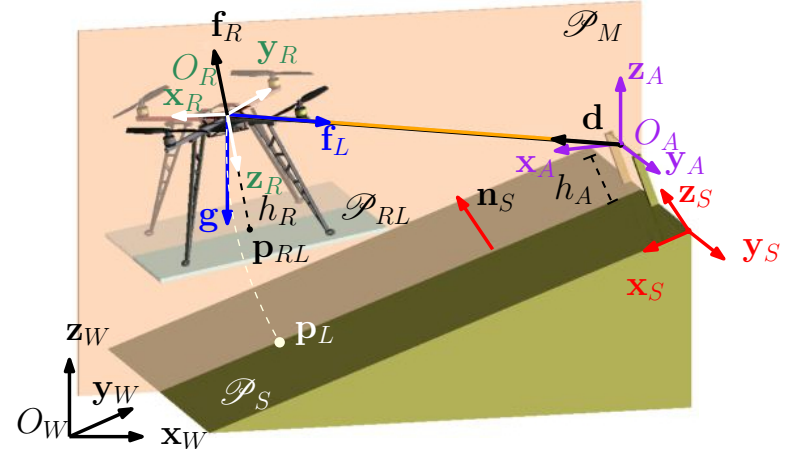

Fig. 1: Representation of the system and its main variables.

$O_{R}$ and $\tau_{R}$ are the coordinates of the 3D control moment expressed in $\mathscr{F}_{R}$. We assume the vehicle equipped with at least three landers whose ending parts form the landers plane $\mathscr{P}_{R L}$. As in the most common case in reality, we assume $\mathbf{z}_{R}$ perpendicular to $\mathscr{P}_{R L}$. Then we define $\mathbf{p}_{R L} \in \mathbb{R}^{3}$ as the projection of $\mathbf{p}_{R}$ on $\mathscr{P}_{R L}$ and $h_{R}=\left\|\mathbf{p}_{R}-\mathbf{p}_{R L}\right\|$.

We assume that the landing/takeoff (LTO) surface is planar in the neighborhood of the desired landing point and it is defined by $\mathscr{P}_{S}:=\left\{\mathbf{p}=\left[\begin{array}{lll}x & y & z\end{array}\right]^{T} \in \mathbb{R}^{3} \mid a x+b y+c z+d=\right.$ $0\}$ where $a, b, c, d \in \mathbb{R}$ are the parameters of the plane. In particular $\mathbf{n}_{S}=\left(1 / \sqrt{a^{2}+b^{2}+c^{2}}\right)[a b c]^{T}$ are the coordinates in $\mathscr{F}_{W}$ of the unit vector normal to $\mathscr{P}_{S}$. Then we define a frame $\mathscr{F}_{S}$ that is rigidly attached to $\mathscr{P}_{S}$, whose axes are $\left\{\mathbf{x}_{S}, \mathbf{y}_{S}, \mathbf{z}_{S}\right\}$. If $\mathbf{n}_{S}=\mathbf{z}_{W}$, i.e., $\mathscr{P}_{S}$ is horizontal, then we set $\left\{\mathbf{x}_{S}, \mathbf{y}_{S}, \mathbf{z}_{S}\right\}=\left\{\mathbf{x}_{W}, \mathbf{y}_{W}, \mathbf{z}_{W}\right\}$. In the others (more interesting) cases, i.e., when $\mathscr{P}_{S}$ is locally inclined, the axes of $\mathscr{F}_{S}$ are set as: $\mathbf{z}_{S}=\mathbf{n}_{S}, \mathbf{y}_{S}=\left(\mathbf{z}_{W} \times \mathbf{z}_{S}\right) /\left\|\mathbf{z}_{W} \times \mathbf{z}_{S}\right\|$ and $\mathbf{x}_{S}=\left(\mathbf{y}_{S} \times\right.$ $\left.\mathbf{z}_{S}\right) /\left\|\mathbf{y}_{S} \times \mathbf{z}_{S}\right\|$. The origin of $\mathscr{F}_{S}, O_{S}$, is taken as any arbitrary position on $\mathscr{P}_{S}$. Fig. 1 gives a schematic representation of the whole system.

\section{Model in Free (Non-tethered) Flight}

The aerial vehicle has six degrees of freedom (DoFs) and its configuration is described by $\mathbf{p}_{R}$ and $\mathbf{R}_{R}$. The well known dynamics of a free-flying VTOL is obtained by solving the Newton-Euler equations at $O_{R}$ :

$$
\begin{aligned}
m_{R} \ddot{\mathbf{p}}_{R} & =-m_{R} g \mathbf{z}_{W}-f_{R} \mathbf{z}_{R} \\
\mathbf{J}_{R} \dot{\boldsymbol{\omega}}_{R} & =\mathbf{J}_{R} \boldsymbol{\omega}_{R} \times \boldsymbol{\omega}_{R}+\boldsymbol{\tau}_{R},
\end{aligned}
$$

where $g$ is the gravitational constant at the landing site. The orientation kinematics is given by $\dot{\mathbf{R}}_{R}=\mathbf{R}_{R} \boldsymbol{\Omega}_{R}$, where $\boldsymbol{\Omega}_{\star}$ is the skew symmetric matrix associated to $\boldsymbol{\omega}_{\star}$.

Model (1) holds as long as the aerial vehicle is not in contact with the surface. In this last case, i.e., $\mathscr{P}_{R L} \equiv \mathscr{P}_{S}$, (1) has to be extended taking into account the reaction force of the surface, denoted by $f_{N} \in \mathbb{R}$, and the static friction force, denoted by $\mathbf{f}_{S} \in \mathbb{R}^{3}$, thus obtaining:

$$
m_{R} \ddot{\mathbf{p}}_{R}=-m_{R} g \mathbf{z}_{W}-f_{R} \mathbf{z}_{R}+f_{N} \mathbf{n}_{S}+\mathbf{f}_{S},
$$

where $f_{N} \geq f_{N}, \mathbf{z}_{S}^{T} \mathbf{f}_{S}=0$ and $\left\|\mathbf{f}_{S}\right\| \leq \overline{f_{S}}$. For a standard surface $\underline{f_{N}}=0$ and $\overline{f_{S}}=\mu f_{N}$ where $\mu \in \mathbb{R}_{>0}$ is the characteristic friction coefficient of the contact between $\mathscr{P}_{R L}$ and $\mathscr{P}_{S}$.

If $\mathscr{P}_{R L}$ and $\mathscr{P}_{S}$ are equipped with an adhesive membrane (as, e.g., a Velcro or a gecko inspired material) then $f_{N} \in \mathbb{R}_{<0}$ is the maximum negative reaction force. In these cases both $\underline{f_{N}}$ and $\overline{f_{S}}$ depend on the adhesive membrane.

\section{Model in Tethered Flight}

Let us consider the case of an aerial vehicle tethered to a fixed point through a link, such as a cable or a chain. One end of the link is attached to the aerial vehicle at $O_{R}$ through a passive 3D spherical joint and the other end is attached to an anchor point $O_{A}$ rigidly attached to the surface. The position of $O_{A}$ is described by $\mathbf{p}_{A} \in \mathbb{R}^{3}$ in $\mathscr{F}_{W}$ and its distance from $\mathscr{P}_{S}$ is given by $h_{A}=\mathbf{z}_{S}^{T}\left(\mathbf{p}_{A}-\mathbf{p}_{L}\right) \in \mathbb{R}_{>0}, \forall \mathbf{p}_{L} \in \mathscr{P}_{S}$.

As in many other works [5] - [9], we assume negligible link mass and inertia with respect to the one of the aerial vehicle and negligible deformations and elasticity. The link length and the intensity of the internal force are denoted by $l \in \mathbb{R}_{\geq 0}$ and $f_{L} \in \mathbb{R}_{>0}$, respectively. The tension of the link is equal to $f_{L}$ when $f_{L}>0$ and $\left\|\left(\mathbf{p}_{R}-\mathbf{p}_{A}\right)\right\|=l$, while it is equal to zero otherwise (slack link). When the link is slack and the aerial vehicle is not in contact with the LTO surface the dynamic model of the system is given by (1)-(2).

On the other hand, when the link is taut $\mathbf{p}_{R} \in \mathscr{S}_{l}\left(\mathbf{p}_{A}\right)=$ $\left\{\mathbf{p} \in \mathbb{R}^{3} \mid \mathbf{p}=\mathbf{p}_{A}+l \mathbf{d}, \forall \mathbf{d} \in \mathrm{S}^{2}\right\}$, where $\mathscr{S}_{l}\left(\mathbf{p}_{A}\right)$ is a sphere of radius $l$ centered on $\mathbf{p}_{A}$, and $\mathbf{d}$ is the unit vector that represents the attitude of the link expressed in $\mathscr{F}_{W}$.

We introduce the frame $\mathscr{F}_{A}=O_{A},\left\{\mathbf{x}_{A}, \mathbf{y}_{A}, \mathbf{z}_{A}\right\}$ defined as $\mathbf{z}_{A}=\mathbf{z}_{W}, \mathbf{y}_{A}=\mathbf{y}_{S}$ and $\mathbf{x}_{A}=\mathbf{y}_{A} \times \mathbf{z}_{A} /\left\|\mathbf{y}_{A} \times \mathbf{z}_{A}\right\| . \mathbf{d}^{A}$ denotes the expression of $\mathbf{d}$ in $\mathscr{F}_{A}$. We parametrize $\mathbf{d}^{A}$ by the elevation angle, $\varphi \in[0,2 \pi]$, and the azimuth angle, $\delta \in\left[-\frac{\pi}{2}, \frac{\pi}{2}\right]$, defined by $\mathbf{d}^{A}=[\cos \delta \cos \varphi-\sin \delta \cos \delta \sin \varphi]^{T}$, where $\delta$ is the angle between $\mathbf{d}^{A}$ and the vertical plane $\left\{\mathbf{x}_{A}, \mathbf{z}_{A}\right\}$, and $\varphi$ is the angle between the projection of $\mathbf{d}^{A}$ on $\left\{\mathbf{x}_{A}, \mathbf{z}_{A}\right\}$ and $\mathbf{x}_{A}$. Thus the translational DoFs of the aerial vehicle reduce to two w.r.t. the free-flight case, and $\mathbf{p}_{R}$ can be described by the generalized coordinates $\mathbf{q}=\left[\begin{array}{ll}\varphi & \delta\end{array}\right]^{T}$ and the parameter $l$.

Since the link is attached to $O_{R}$, the rotational dynamics of the vehicle is independent of the translational one and it is equal to (2). We retrieve the dynamics of $\mathbf{q}$ with the Newton-Euler method applying the balance of forces at $O_{R}$ :

$$
m_{R} \ddot{\mathbf{p}}_{R}=-m_{R} g \mathbf{z}_{W}-f_{R} \mathbf{z}_{R}-f_{L} \mathbf{d},
$$

where $\ddot{\mathbf{p}}_{R}$ is obtained differentiating twice $\mathbf{p}_{R}=\mathbf{p}_{A}+l \mathbf{R}_{A} \mathbf{d}^{A}$ :

$$
\ddot{\mathbf{p}}_{R}=\mathbf{R}_{A}\left(\dot{\mathbf{J}}_{\mathbf{q}} \dot{\mathbf{q}}+\mathbf{J}_{\mathbf{q}} \ddot{\mathbf{q}}\right), \quad \mathbf{J}_{\mathbf{q}}=\left[\begin{array}{cc}
-l \cos \delta \sin \varphi & -l \cos \varphi \sin \delta \\
0 & -l \cos \delta \\
l \cos \delta \cos \varphi & -l \sin \delta \sin \varphi
\end{array}\right],
$$

where $\mathbf{R}_{A} \in \mathbb{R}^{3 \times 3}$ is the rotation matrix from $\mathscr{F}_{A}$ to $\mathscr{F}_{W}$. Equations (2) and (4) fully describe the dynamics of the system when the link is taut.

Similarly to the non-tethered case, when the robot is tethered and in contact with the surface, the model (4) is extended taking into account the forces $f_{N} \in \mathbb{R}$ and $f_{S} \in \mathbb{R}$ :

$$
m_{R} \ddot{\mathbf{p}}_{R}=-m_{R} g \mathbf{z}_{W}-f_{R} \mathbf{z}_{R}-f_{L} \mathbf{d}+f_{N} \mathbf{n}_{S}+\mathbf{f}_{S}
$$

\section{CONDITIONS FOR LANDING AND TAKE-OFF MANEUVERS}

In the following we define and analyze the problem of landing on $\mathscr{P}_{S}$ at a desired landing position $\mathbf{p}_{L}^{\star} \in \mathscr{P}_{S}$. Analogous conditions can be drawn for the takeoff problem, which are omitted here for brevity. Denoting with $t_{L} \in \mathbb{R}_{>0}$ 
the landing time, a correct and robust landing is such if the following conditions are satisfied:

Cond. 1) $\mathbf{p}_{R L}$ converges to $\mathbf{p}_{L}^{\star}$, i.e., $\mathbf{p}_{R L}\left(t_{L}\right)=\mathbf{p}_{L}^{\star} \in \mathscr{P}_{S}$;

Cond. 2) the robot orientation has to be such that $\mathscr{P}_{R L}$ and $\mathscr{P}_{S}$ are parallel, i.e., $\mathbf{z}_{R}\left(t_{L}\right)=\mathbf{z}_{R}^{\star}=-\mathbf{z}_{S}$, in order to have the robot perfectly in contact with the surface;

Cond. 3) the vehicle has to reach this configuration with almost zero kinetic energy in order to avoid hard impacts, i.e., at time $t_{L}{ }^{-}$, immediately before of touching the surface, it has to be that $\dot{\mathbf{p}}_{R}\left(t_{L}{ }^{-}\right)=\mathbf{0}$ and $\boldsymbol{\omega}_{R}\left(t_{L}{ }^{-}\right)=\mathbf{0}$;

Cond. 4) all the accelerations should be also zero at $t_{L}{ }^{-}$, i.e., $\ddot{\mathbf{p}}_{R}\left(t_{L}{ }^{-}\right)=\mathbf{0}$ and $\dot{\boldsymbol{\omega}}_{R}\left(t_{L}{ }^{-}\right)=\mathbf{0}$, thus obtaining a smooth and gentle maneuver;

Definition 1 (Inclined hovering). The system is said in inclined hovering if $\mathbf{z}_{R} \neq-\mathbf{z}_{W}$ and Cond. 3, and 4 coexist.

Cond. 5) after the conclusion of the landing maneuver, at time $t_{L}{ }^{+}$, when the robot is in contact with the surface, $\mathbf{p}_{L}^{\star}$ has to be a stable position, i.e., zero velocity and acceleration. This condition prevents the robot to fly away from the surface or to slide down on it when the motors are switched off.

Remark 1. At time $t_{L}{ }^{-}$the robot is not yet in contact with the surface and the flying model has to be used to describe the system (equations $(1,2)$ or $(4,2)$ ). On the contrary, at time $t_{L}{ }^{+}$ the vehicle is in contact with the surface thus equations (3) or (5) have to be used.

Notice that the Cond. 4, although not strictly necessary, lets the vehicle approach the surface in a static equilibrium condition, passing from flight to contact very smoothly and in a more robust way with respect to model uncertainties.

If, due to the characteristics of the system, Cond. 4 is not attainable, the landing can still be done but when at time $t_{L}{ }^{+}$the vehicle touches the surface, one has to find the way (e.g., turning off the motors as quickly as possible and using a Velcro system) to immediately pass in a stable condition in order to remain in contact with the surface without flying away or sliding on it (Cond. 5). Nevertheless, this could be not possible for some surfaces without the use of a tether or a Velcro-like solution.

\section{ANALYSis AND COMPARISON OF FREE-FLIGHT VS. TETHERED MANEUVERS FOR LANDING/TAKEOFF}

\section{A. Free Flight Method}

Replacing the conditions $\mathbf{z}_{S}=\mathbf{z}_{W}$ and $\ddot{\mathbf{p}}_{R}\left(t_{L}{ }^{-}\right)=\mathbf{0}$ in (1), it is clear that the only case in which Cond. 4 holds is when $\mathscr{P}_{S}$ is horizontal. In all the other cases $\ddot{\mathbf{p}}_{R}\left(t_{L}{ }^{-}\right) \neq \mathbf{0}$, which means that the aerial vehicle cannot approach the surface in a fully stable condition.

For the Cond. 5, imposing $\ddot{\mathbf{p}}_{R}\left(t_{L}{ }^{+}\right)=\mathbf{0}$ in (3) and projecting the two sides of (3) on $\mathscr{F}_{S}$, we obtain

$$
f_{N}=m_{R} g \mathbf{z}_{S}^{T} \mathbf{z}_{W}+f_{R}, \quad \mathbf{x}_{S}^{T} \mathbf{f}_{S}=m_{R} g \mathbf{x}_{S}^{T} \mathbf{z}_{W}, \quad \mathbf{y}_{S}^{T} \mathbf{f}_{S}=0 .
$$

The first two conditions of (6) let us determine which is the maximum thrust at time $t_{L}{ }^{+}$and the maximum slope to have Cond. 5 fulfilled, i.e.:

$$
f_{R}\left(t_{L}{ }^{+}\right) \leq m_{R} g \mathbf{z}_{S}^{T} \mathbf{z}_{W}-\underline{f_{N}} \quad \text { and } \quad \mathbf{x}_{S}^{T} \mathbf{z}_{W} \leq \overline{f_{S}} /\left(m_{R} g\right) .
$$

Thus, one can land on any point of $\mathscr{P}_{S}$ only if (7) holds, restricting the set of admissible slopes.
Assuming that the surface fulfills (7), we now investigate how to reach it, and in particular how to achieve the first three conditions. In the less interesting case of a horizontal surface, one can simply follow a trajectory along $\mathbf{z}_{W}$ in hovering condition to reach $\mathbf{p}_{L}^{\star}$ with zero velocities and accelerations. In the more interesting case of a sloped surface this is a very challenging problem due to the underactuation of the vehicle. From the theory it is well known that the system is differentially flat with respect to $\mathbf{p}_{R}$ and the rotation around $\mathbf{z}_{R}$ [4]. Therefore one can track any desired position trajectory, $\mathbf{p}_{R}^{d}(t)$, such that $\mathbf{p}_{R L}\left(t_{L}\right)=\mathbf{p}_{L}^{\star}$ and $\dot{\mathbf{p}}_{R}\left(t_{L}{ }^{-}\right)=\mathbf{0}$, but the orientation of the vehicle along the trajectory is exactely determined by $\mathbf{p}_{R}^{d}(t)$ and its derivatives. Thus it is not possible to control the attitude independently of the position trajectory. The classical method to overcome this issue is to use a state-to-state planner like, e.g., the ones presented in [3] slightly modified, that gives a particular position trajectory $\mathbf{p}_{R}^{d}(t)$ that satisfies Conds 1,2 and 3 .

Assuming that the non-easy planning problem is solved, one could use different types of controllers, as the ones in [1], [2], to track the planned trajectory. Nevertheless, these methods lack in general of robustness since small tracking errors could lead, e.g., to miss the target or to crash on it if the velocity is not well tracked. Furthermore, a precise model and an accurate and high-rate state estimation are needed.

\section{B. Tethered Method}

In this section we show that the tethered method overcomes the limits of free-flight (in particular, the impossibility to satisfy Cond. 4 for sloped surfaces, which guaranties a safer landing maneuver) thanks to the inclined equilibria.

For the tethered method the landing position must belong to $\mathscr{S}_{l}\left(\mathbf{p}_{A}\right) \cap \mathscr{P}_{S}$. We then first investigate which are the points in this set that satisfy Cond. 4. Consider a generic point $\mathbf{p}_{L} \in \mathscr{S}_{l}\left(\mathbf{p}_{A}\right) \cap \mathscr{P}_{S}$. From simple geometry we have

$$
\mathbf{d}=\left(\mathbf{p}_{L}-\mathbf{p}_{A}+h_{R} \mathbf{z}_{S}\right) / l .
$$

Since $\mathbf{p}_{A}, l$ and $h_{R}$ are given parameters, finding the $\mathbf{p}_{L}$ that satisfies Cond. 4 is equivalent to find the $\mathbf{d}$ that satisfies the same condition. Projecting both sides of (8) on $\mathbf{z}_{S}^{T}$ we obtain

$$
\mathbf{z}_{S}^{T} \mathbf{d}=\left(h_{R}+\mathbf{z}_{S}^{T}\left(\mathbf{p}_{L}-\mathbf{p}_{A}\right)\right) / l=\left(h_{R}-h_{A}\right) / l .
$$

Then, in order to fulfill Cond. 4, let us project both sides of (4) on the plane $\left\{\mathbf{x}_{S}, \mathbf{y}_{S}\right\}$, and set $\ddot{\mathbf{p}}_{R}=\mathbf{0}$, thus obtaining

$$
f_{L} \mathbf{P}_{x y}^{S} \mathbf{d}=-m_{R} g \mathbf{P}_{x y}^{S} \mathbf{z}_{W},
$$

where $\mathbf{P}_{x y}^{S}=\left[\begin{array}{ll}\mathbf{x}_{S} & \mathbf{y}_{S}\end{array}\right]^{T}$. Equation (10) implies that $\mathbf{P}_{x y}^{S} \mathbf{d}$ is parallel to $\mathbf{P}_{x y}^{S} \mathbf{z}_{W}$. Since $f_{L} \geq 0$ and $m_{R} g>0$, we obtain

$$
\left(\mathbf{P}_{x y}^{S} \mathbf{d}\right) /\left\|\mathbf{P}_{x y}^{S} \mathbf{d}\right\|=-\left(\mathbf{P}_{x y}^{S} \mathbf{z}_{W}\right) /\left\|\mathbf{P}_{x y}^{S} \mathbf{z}_{W}\right\|=: \mathbf{z}_{W}^{S_{x y}} .
$$

Notice that (11) requires ${ }^{1}\left\|\mathbf{P}_{x y}^{S} \mathbf{z}_{W}\right\| \neq 0$ and $\left\|\mathbf{P}_{x y}^{S} \mathbf{d}\right\| \neq 0$. The latter inequality implies also that $\left(h_{R}-h_{A}\right) \neq l$. From (9)(11) and applying some simple geometry we obtain

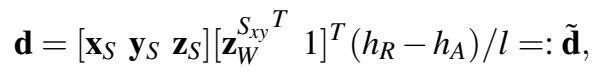

${ }^{1}$ When $\left\|\mathbf{P}_{x y}^{S} \mathbf{z}_{W}\right\|=0$ the surface is horizontal and any $\mathbf{d}$ such that (9) holds, satisfies Cond. 4. In this condition, one can still land with the tethered configuration keeping $f_{L}=0$ and using the same method for free flight. 


\begin{tabular}{|c|c|c|c|c|}
\hline Method for landing & \multicolumn{2}{|r|}{ Free Flight } & \multicolumn{2}{|c|}{ Tethered Flight } \\
\hline Fulfillment of Conditions & All & All but Cond. $4\left(\ddot{\mathbf{p}}_{R}\left(t_{L}^{-}\right) \neq \mathbf{0}\right)$ & All & All but Cond. $4\left(\ddot{\mathbf{p}}_{R}\left(t_{L}{ }^{-}\right) \neq \mathbf{0}\right)$ \\
\hline Surface orientations & $\mathbf{z}_{S}=\mathbf{z}_{W}$ & $\mathbf{x}_{S}^{T} \mathbf{z}_{W} \leq \overline{f_{S}} /\left(m_{R} g\right)$ & any & $\left|-m_{R} g \mathbf{y}_{S}^{T} \mathbf{d}\left(\mathbf{x}_{S}^{T} \mathbf{z}_{W} \mathbf{x}_{S}^{T} \mathbf{d}\right)\right| \leq \overline{f_{S}}$ \\
\hline Anchor positions, $\mathbf{p}_{A}$ & - & - & any & any \\
\hline Landing positions $\mathbf{p}_{L}$ & any & any & $\mathbf{p}_{L}=\mathbf{p}_{A}+l \tilde{\mathbf{d}}-h_{R} \mathbf{z}_{S}$ & $\mathbf{p}_{L} \in \mathscr{S}_{l}\left(\mathbf{p}_{A}\right) \cap \mathscr{P}_{S}$ \\
\hline Maximum thrust at time $t_{L}^{+}$ & $f_{R} \leq m_{R} g-f_{N}$ & $f_{R} \leq m_{R} g \mathbf{z}_{S}^{T} \mathbf{z}_{W}-\underline{f_{N}}$ & $f_{R}\left(t_{L}^{+}\right) \leq \overline{f_{R}}$ & $f_{R}\left(t_{L}^{+}\right) \leq \overline{f_{R}}$ \\
\hline Pros & \multicolumn{2}{|l|}{ Simple system } & \multicolumn{2}{|c|}{$\begin{array}{l}\text { Possibility to perform the maneuver reaching a sta- } \\
\text { ble equilibria condition; a planner is not required; } \\
\text { robustness to model uncertainties and tracking errors }\end{array}$} \\
\hline Cons & \multicolumn{2}{|c|}{$\begin{array}{l}\text { Not feasible for every slope; it requires: a planner, } \\
\text { high tracking accuracy, precise state estimation and } \\
\text { knowledge of the model (very low robustness) }\end{array}$} & \multicolumn{2}{|c|}{$\begin{array}{l}\text { Need of a method to pass from free-flight condition } \\
\text { to tethered one }\end{array}$} \\
\hline
\end{tabular}

TABLE I: Characteristics of free-flight and tethered configuration for the landing problem. Analogous conditions hold for the take-off.

where $\tilde{\mathbf{d}}$ is defined as the (unique) $\mathbf{d}$ for which Cond. 4 is fulfilled. This proves that, given the parameters of the system, $\mathbf{p}_{A}, l$ and $h_{R}$, it exists a (unique) $\tilde{\mathbf{p}}_{L}=\mathbf{p}_{A}+l \tilde{\mathbf{d}}-h_{R} \mathbf{z}_{S}$, for which Cond. 4 is respected.

Remark 2. The use of a tether creates the conditions to approach or depart from a sloped surface in a stable equilibria condition (inclined hovering), i.e., in a more robust and safer way. In fact, using the tether it exists a landing position in which one can land in inclined hovering for any sloped surface (in free-flight this position exists only for horizontal surfaces). Moreover, given any desired landing position $\mathbf{p}_{L}^{\star} \in$ $\mathscr{P}_{S}$, one can always fulfill Cond. 4 setting $h_{A} \neq h_{R}-l$ and

$$
\mathbf{p}_{A}=\mathbf{p}_{L}^{\star}+h_{R} \mathbf{z}_{S}-l \tilde{\mathbf{d}}:=\tilde{\mathbf{p}}_{A} .
$$

Compliance with Cond. 5: If $\left(\mathbf{x}_{S}^{T} \mathbf{z}_{W}\right)\left(\mathbf{x}_{S}^{T} \mathbf{d}\right)<0$, then a solution of (5) for $\ddot{\mathbf{p}}_{R}\left(t_{L}^{+}\right)=\mathbf{0}$ is

$$
\begin{array}{ll}
f_{L}=-\frac{m_{R} g \mathbf{x}_{S}^{T} \mathbf{z}_{W}}{\mathbf{x}_{S}^{T} \mathbf{d}}, & f_{N}=m_{R} g \mathbf{z}_{S}^{T} \mathbf{z}_{W}-f_{R}+f_{L} \mathbf{z}_{S}^{T} \mathbf{d}, \\
\mathbf{y}_{S}^{T} \mathbf{f}_{S}=f_{L} \mathbf{y}_{S}^{T} \mathbf{d}, & \mathbf{x}_{S}^{T} \mathbf{f}_{S}=0 .
\end{array}
$$

In this case the tension is always positive and, from the conditions on $f_{N}$ and $\mathbf{f}_{S}$ in (14), we can determine which is the maximum thrust intensity at time $t_{L}{ }^{+}$and the maximum slope of the surface to respect the Cond. 5, i.e.,

$$
\begin{array}{r}
f_{R}\left(t_{L}{ }^{+}\right) \leq m_{R} g \mathbf{z}_{S}^{T} \mathbf{z}_{W}\left(1-\left(\mathbf{z}_{S}^{T} \mathbf{d} / \mathbf{x}_{S}^{T} \mathbf{d}\right)\right)-\underline{f_{N}}=: \overline{f_{R}} \\
\left|-m_{R} g \mathbf{y}_{S}^{T} \mathbf{d}\left(\mathbf{x}_{S}^{T} \mathbf{z}_{W} / \mathbf{x}_{S}^{T} \overline{\mathbf{d}}\right)\right| \leq \overline{f_{S}} .
\end{array}
$$

If $\mathbf{d}=\tilde{\mathbf{d}}$ then the condition (16) holds for any surface. In the opposite case of $\left(\mathbf{x}_{S}^{T} \mathbf{z}_{W}\right)\left(\mathbf{x}_{S}^{T} \mathbf{d}\right) \geq 0, f_{L}=0$ and the conditions in (7) have to be respected.

Table I summarizes all the previous results. How to accomplish Cond.1, 2 and 3 will be presented in Sec. V. Notice that with the tethered method we can achieve all the landing conditions for any surface and any desired landing position by properly choosing the anchor point.

\section{Controller for Tethered LANDing/TAKeofF}

To land/takeoff on/from $\mathscr{P}_{S}$ in $\mathbf{p}_{L}^{\star}$, respecting all the objectives of Sec. III exploiting the tether we use a tracking control approach based on the flatness of the system.

\section{A. Differential flatness}

A system is called differentially flat with respect to an output, named flat output, if its state and inputs can be written as an algebraic function of the flat output and a finite number of its derivatives. In this section we demonstrate that $\mathbf{y}^{\vartheta_{A}}=$ $\left[\begin{array}{llll}y_{1}^{\vartheta_{A}} & y_{2}^{\vartheta_{A}} & y_{3}^{\vartheta_{A}} & y_{4}^{\vartheta_{A}}\end{array}\right]^{T}=\left[\begin{array}{llll}\varphi & \delta & \vartheta_{A} & \psi_{R}\end{array}\right]^{T} \in \mathbb{R}^{4}$ is a flat output for the system $(4,2)$ where $\vartheta_{A}$ and $\psi_{R}$ represent the rotations of $\mathscr{F}_{R}$ around $\mathbf{y}_{A}$ and $\mathbf{z}_{R}$, respectively.

First of all, let us consider the output $\mathbf{y}^{f_{L}}=$ $\left[\begin{array}{llll}y_{1}^{f_{L}} & y_{2}^{f_{L}} & y_{3}^{f_{L}} & y_{4}^{f_{L}}\end{array}\right]^{T}=\left[\begin{array}{llll}\varphi & \delta & f_{L} & \psi_{R}\end{array}\right]^{T} \in \mathbb{R}^{4}$ that, as demonstrated in [10] (and previoulsy in [7] for a similar system) is another flat output. Now, in order to demonstrate that $\mathbf{y}^{\vartheta_{A}}$ is also a flat output we show that there exists a bijective map between $\mathbf{y}^{\vartheta_{A}}$ and $\mathbf{y}^{f_{L}}$. From the definition of $\vartheta_{A}$ we can write

$$
-\left[\begin{array}{ll}
\sin \vartheta_{A} & \cos \vartheta_{A}
\end{array}\right]^{T}=\left(\mathbf{P}_{x z}^{A} \mathbf{z}_{R}\right) /\left\|\mathbf{P}_{x z}^{A} \mathbf{z}_{R}\right\|=\overline{\mathbf{z}}_{R x z}^{A},
$$

where $\mathbf{P}_{x z}^{A}=\left[\begin{array}{ll}\mathbf{x}_{A} & \mathbf{z}_{A}\end{array}\right]^{T}$ is the projector on $\left\{\mathbf{x}_{A}, \mathbf{z}_{A}\right\}$. The map from $\mathbf{y}^{f_{L}}$ to $\mathbf{y}^{\vartheta_{A}}$ and their derivatives, is simply given by the flatness of the system w.r.t. $\mathbf{y}^{f_{L}}$. In fact, given $\mathbf{y}^{f_{L}}$ and its derivatives one can compute state and inputs. Then from equation (17) it is easy to compute $\mathbf{y}^{\vartheta_{A}}$ and its derivatives.

Regarding the opposite sense of the map, i.e., from $\mathbf{y}^{\vartheta_{A}}$ to $\mathbf{y}^{f_{L}}$, the map is immediate for $\mathbf{q}$ and $\psi_{R}$. Then we can retrieve $f_{L}$ from $\mathbf{y}^{\vartheta_{A}}$ and its derivatives projecting both sides of equation (4) on the plane $\left\{\mathbf{x}_{A}, \mathbf{z}_{A}\right\}$, and after some algebra:

$$
f_{L}=\left[\begin{array}{ll}
1 & 0
\end{array}\right] \mathbf{T}^{-1} \mathbf{a}=g_{\vartheta_{A}}^{f_{L}}\left(\mathbf{y}^{\vartheta_{A}}, \dot{\mathbf{y}}^{\vartheta_{A}}, \ddot{\mathbf{y}}^{\vartheta_{A}}\right)
$$

where $\mathbf{a}\left(\mathbf{y}^{\vartheta_{A}}, \dot{\mathbf{y}}^{\vartheta_{A}}, \ddot{\mathbf{y}}^{\vartheta_{A}}\right)=-m_{R} g \mathbf{P}_{x z}^{A}\left(\ddot{\mathbf{p}}_{R}+g \mathbf{z}_{W}\right)$ and $\mathbf{T}\left(\mathbf{y}^{\vartheta_{A}}\right)=$ $\left[\mathbf{P}_{x z}^{A} \mathbf{d} \overline{\mathbf{z}}_{R x z}^{A}\right]$ is invertible if and only if $\mathbf{d}^{T} \mathbf{z}_{R} \neq 0$. Finally, to retrieve the derivatives of $f_{L}$ one can simply differentiate (18) w.r.t. time. This proves that between $\mathbf{y}^{f_{L}}$ and $\mathbf{y}^{\vartheta_{A}}$, and their derivatives, there is a bijective map. With this result, state and inputs of the system can be written as an algebraic function of $\mathbf{y}^{\vartheta_{A}}$ and its derivatives proving that $\mathbf{y}^{\vartheta_{A}}$ is a flat output.

\section{B. Hierarchical Controller}

In this section we design a controller based on a hierarchical technique in order to control $\mathbf{y}^{\vartheta_{A}}$ and track a feasible desired trajectory $\mathbf{y}^{\vartheta_{A} d}(t)$. Similar methods were already successfully applied for both free-flying [2] and tethered 
vehicles to only control the position [9] or the attitude [8]. Those methods can not be directly applied to our case since our system and purposes are different.

Our method is based on a cascade of two controllers. The first, the outer-loop controller, controls the translational dynamics assuming as control inputs thrust and attitude. The second, the inner-loop controller, controls the rotational dynamics tracking the desired attitude computed by the outer-loop. Fig. 2 gives a schematic representation.

In order to steer the generalized coordinates along a desired trajectory $\mathbf{q}^{d}(t)$ we define $\ddot{\mathbf{q}}^{\star}=\ddot{\mathbf{q}}^{d}+k_{\mathbf{q}}^{D}\left(\dot{\mathbf{q}}^{d}-\dot{\mathbf{q}}\right)+$ $k_{\mathbf{q}}^{P}\left(\dot{\mathbf{q}}^{d}-\dot{\mathbf{q}}\right)$, the desired acceleration that lets $\mathbf{q}$ follow the desired configuration $\mathbf{q}^{d}$ using a PD strategy, where $k_{\mathbf{q}}^{P}, k_{\mathbf{q}}^{D} \in$ $\mathbb{R}^{+}$are the classical gains. Then, using (18) we define by $f_{L}^{\star}(t)=g_{\vartheta_{A}}^{f_{L}}\left(\mathbf{y}^{\vartheta_{A} d}(t)\right)$ the nominal stress that $\mathbf{y}^{\vartheta_{A} d}(t)$ requires. Then, in order to obtain $\ddot{\mathbf{q}}^{\star}$, considering as input of the translational dynamics the thrust vector $\mathbf{f}_{R}=-f_{R} \mathbf{z}_{R}$, we define the control law by inverting (4) as

$$
\mathbf{f}_{R}^{\star}=-m_{R} g \mathbf{Z}_{W}-g_{\vartheta_{A}}^{f_{L}}\left(\mathbf{y}^{\vartheta_{A} d}\right) \mathbf{d}-m_{R} \mathbf{R}_{A}\left(\dot{\mathbf{J}}_{\mathbf{q}} \dot{\mathbf{q}}+\mathbf{J}_{\mathbf{q}} \ddot{\mathbf{q}}^{\star}\right) .
$$

From (19) the intensity of the thrust and the desired direction of $\mathbf{z}_{R}$ are computed by $f_{R}=\left\|\mathbf{f}_{R}^{\star}\right\|$ and $\mathbf{z}_{R}^{\star}=\mathbf{f}_{R}^{\star} /\left\|\mathbf{f}_{R}^{\star}\right\|$, respectively. The vector $\mathbf{z}_{R}^{\star}$ together with $\psi_{R}^{d}$ univocally define the desired attitude of the vehicle $\mathbf{R}_{R}^{\star} \in S O(3)$.

To track $\mathbf{R}_{R}^{\star}$ we first define the desired angular acceleration using a PD strategy by $\dot{\boldsymbol{\omega}}_{R}^{\star}=-k_{\omega}^{D} \boldsymbol{\omega}_{R}+k_{\omega}^{P} \mathbf{e}_{R}$, where $k_{\omega}^{P}, k_{\omega}^{D} \in$ $\mathbb{R}^{+}$and $\mathbf{e}_{R} \in \mathbb{R}^{3}$ is the error related to the attitude computed by $\left[\mathbf{e}_{R}\right]_{\times}=-\frac{1}{2}\left(\mathbf{R}_{R}^{\star T} \mathbf{R}_{R}-\mathbf{R}_{R}^{T} \mathbf{R}_{R}^{\star}\right)$. Finally, from (2) the input torque is computed as $\boldsymbol{\tau}_{R}=-\mathbf{J}_{R} \boldsymbol{\omega}_{R} \times \boldsymbol{\omega}_{R}+\mathbf{J}_{R} \dot{\boldsymbol{\omega}}_{R}^{\star}$.

If the inner-loop is sufficiently faster than the outer-loop, the asymptotic convergence of $\mathbf{y}^{\vartheta_{A}}$ to $\mathbf{y}^{\vartheta_{A} d}$ is guaranteed.

\section{Computation of the Desired Output $\mathbf{y}^{\vartheta_{A} d}$}

We assume that in a preliminary phase the vehicle has been tethered to the anchor point $\mathbf{p}_{A}$ defined in (13), such that $\mathbf{p}_{L}^{\star}=\tilde{\mathbf{p}}_{L}$. To accomplish the landing maneuvers, the final

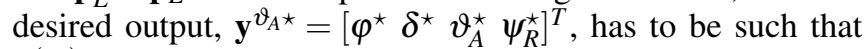
$\mathbf{x}\left(t_{L}\right)=\tilde{\mathbf{x}}$, where $\tilde{\mathbf{x}}$ fulfills the Cond.1 -4 . Since $\mathbf{p}_{R L}$ and $\mathbf{z}_{R}$ are independent of $\psi_{R}, \psi_{R}^{\star}$ can be chosen arbitrarily. From the parametrization of $\mathbf{d}$ and (17) we get

$$
\begin{array}{r}
\varphi^{\star}=\operatorname{atan} 2\left(\mathbf{z}_{A}^{T} \tilde{\mathbf{d}}, \mathbf{x}_{A}^{T} \tilde{\mathbf{d}}\right), \quad \vartheta_{A}^{\star}=\operatorname{atan} 2\left(-\mathbf{x}_{A}^{T} \mathbf{z}_{R}^{\star},-\mathbf{z}_{A}^{T} \mathbf{z}_{R}^{\star}\right) \\
\delta^{\star}=\operatorname{atan} 2\left(\mathbf{y}_{A}^{T} \tilde{\mathbf{d}}, \sqrt{\left(\mathbf{z}_{A}^{T} \tilde{\mathbf{d}}\right)^{2}+\left(\mathbf{x}_{A}^{T} \tilde{\mathbf{d}}\right)^{2}}\right) .
\end{array}
$$

Therefore, if $\mathbf{p}_{A}$ is chosen such that $\mathbf{p}_{L}^{\star}=\tilde{\mathbf{p}}_{L}$, then steering $\mathbf{y}^{\vartheta_{A}}$ to $\mathbf{y}^{\vartheta_{A^{\star}}}$ is sufficient to steer $\mathbf{p}_{R L}$ and $\mathbf{z}_{R}$ to $\mathbf{p}_{L}^{\star}$ and $\mathbf{z}_{R}^{\star}$, respectively. Using splines we can generate a simple sufficiently smooth trajectory $\mathbf{y}^{\vartheta_{A} d}(t)$ from the initial state to $\mathbf{y}^{\vartheta_{A}}$ to fulfill all the objectives of Sec. III, and then track it with the controller presented previously without the use of a planner. Thanks to the fact that the final state is a static equilibria (an inclined hovering), the success of the maneuver does not depend on precise tracking or exact knowledge of the environment and the robot model. In fact, the feedback part of the controllers will provide to the system the needed compliance to successfully fulfill the task.

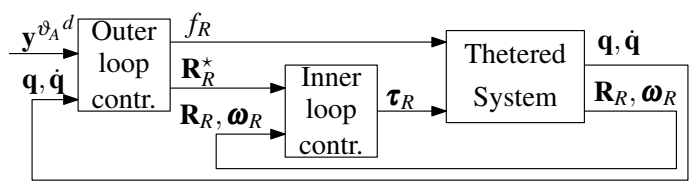

Fig. 2: Schematic representation of the control law.
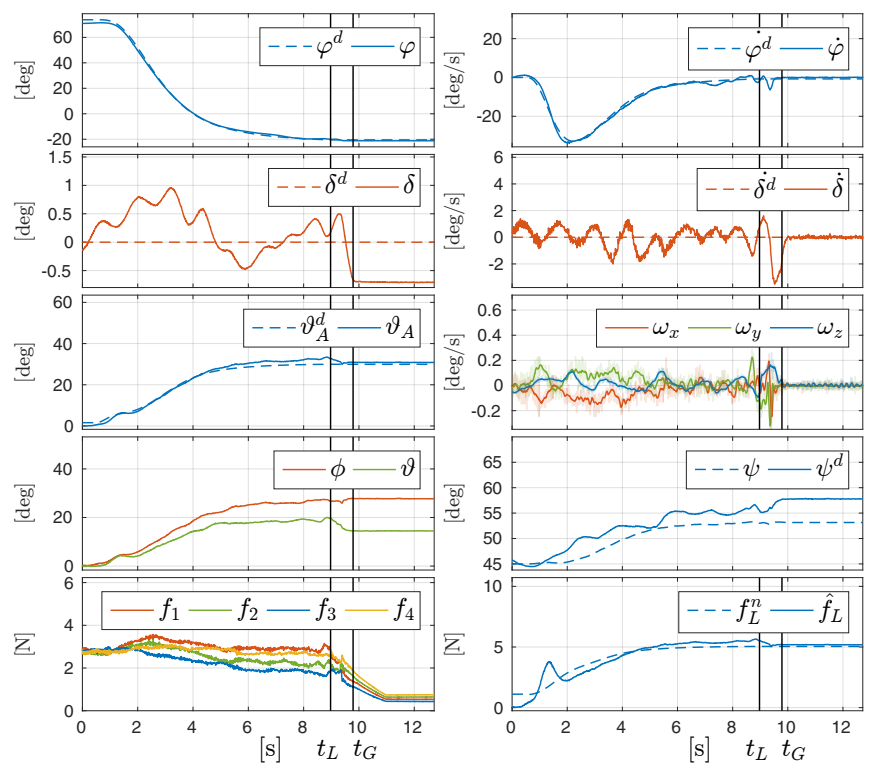

Fig. 3: Tracking of the desired landing maneuver. $f_{L}^{n}$ is the nominal stress given by the particular trajectory, whereas $\hat{f}_{L}$ is the estimated stress from the knowledge of the model, state and inputs. The force produced by the $i$-th propeller is denoted with $f_{i}$ with $i=1 \ldots 4$.

\section{EXPERIMENTAL VALIDATION}

In this section we show the main results of an experimental campaign that validates the efficacy of our method for the problem of landing (and takeoff) on a sloped surface.

The vehicle for our experiments is a Mikrokopter quadrotor weighting about $1[\mathrm{Kg}]$ and having a maximum thrust for each propeller of $6[\mathrm{~N}]$. It is equipped with a light cable ending with a triple hook at its extremity. The other end of the link is attached to the vehicle as close as possible to its CoM. The link has a length of $1[\mathrm{~m}]$ and a mass of less than $0.01[\mathrm{Kg}]$, thus negligible w.r.t. the vehicle one.

The goal of the experiment is to automatically land the robot on a surface, tilted of $\left.30^{\circ}\right]$. The maneuver starts with the vehicle in free-flight and consists of five phases (Fig. 4):

(a) approach to the anchor point with the hook,

(b) hooking of the horizontal cable,

(c) stretching of the anchor,

(d) tracking of the desired trajectory $\mathbf{y}^{\vartheta_{A} d}(t)$ for landing,

(e) turning-off of the propellers after the landing.

Although not necessary, the global maneuver is done moving on the plane $\mathscr{P}_{M}$ (Fig. 1), in order to simplify the phases (a-c). During phase (b), to hook the free extremity of the link to the structure, we placed a horizontal slack cable coincident with the anchor point $\mathbf{p}_{A}$ (a priori decided). A simple maneuver lets the vehicle hook the horizontal cable resulting tethered to the anchor point, as it is shown in Fig. 4.

The controller, fully implemented in Matlab-Simulink, sends the desired rotational speed of each propeller at a frequency of $500[\mathrm{~Hz}]$. In order to retrieve the state of the 




Fig. 4: Sequence of images of a real experiment (see attached video). On the left bottom corner we show a zoom of the anchor point and the hook circled in red. The vehicle is attached by a cable to a pole from the top for security reasons and to connect the robot to the PC by a serial cable. Notice from Fig. (d) that this cable is always slack and does not perturb the motion of the robot.

system we use a motion capture system to measure the position and the yaw angle of the vehicle with a frequency of $120[\mathrm{~Hz}]$. The linear velocity is computed by the numerical derivation. Notice that, when the link is taut, the motion capture system emulates two encoders that measure $\varphi$ and $\delta$. Finally we use an on-board IMU (accelerometer and gyroscope) to complete the measurement of the attitude, estimating the remaining rotation, and the angular rate, $\boldsymbol{\omega}_{R}$.

During phases $(\mathrm{a}-\mathrm{c})$ the vehicle is controlled by a classical near hovering controller. To make the link taut we simply give as reference a position that lies outside the sphere $\mathscr{S}_{l}\left(\mathbf{p}_{A}\right)$. As soon as, at time $t_{0}$, the link is taut (detectable using a threshold in the position error) the controller presented in Sec. V is activated and a simple trajectory $\mathbf{y}^{\vartheta_{A} d}(t)$ is used as reference. Given the parameters of the system, in order to achieve the landing conditions, from Sec. IV-B it results that: $\left.\varphi^{\star}=-21.3\left[^{\circ}\right], \delta^{\star}=0\left[^{\circ}\right], \vartheta_{A}^{\star}=30^{\circ}\right]$. During the maneuver, $\psi_{R}^{\star}$ is set such that the frame of the vehicle is turned by $\left.45^{\circ}\right]$ with respect to the link. Since the link is not perfectly attached to $O_{R}$, the extra torques created by the tension on the robot when the cable is taut are properly compensated, thus improving the tracking performances.

The results of the experiment are shown in Fig. 3 and 4. At time $t_{L}$ the surface is successfully reached and at time $t_{G}$ the motors are turned off completing the maneuver. Although the model of the system was not very accurate, in particular w.r.t. the inertia of the vehicle and the slope of the surface, the full landing maneuver was executed, respecting all the conditions of Sec. III, thus proving the validity and the robustness of the method. Looking at the tracking performances, one can see that the desired trajectory is well tracked, except for some errors due to noisy measurements and model uncertainties.

For additional results including the takeoff we refer the interested reader to the attached video and to the report [10].

\section{CONCLUSION}

Considering a VTOL aerial vehicle, in this manuscript we faced the problem of landing and takeoff on a sloped surface. The problem was analyzed for both free-flight and tethered method showing that the use of a tether increases the stable equilibria configurations allowing to perform the maneuver in a safe and more robust way. Focusing on the tethered system we proved the differential flatness, and we designed a controller to track the position of the vehicle and part of its orientation to perform the landing and takeoff maneuvers without the need of a planner that generates a complex perching maneuver. The method was finally validated through real experiments that show the ability of the proposed method to perform the landing and takeoff maneuvers from sloped surfaces in a safe and robust way.

In the future we plan to work on the use of only on-board sensors like inertial and cameras, and to use sensor-based calibration methods as, e.g., in [11], in order to retrieve the system parameters from the scratch. Extension to multi-robot systems as in [12] will be also considered.

\section{ACKNOWLEDGEMENTS}

We thank Anthony Mallet (LAAS-CNRS) for his contribution in the software architecture of the experiments.

\section{REFERENCES}

[1] D. Cabecinhas, R. Naldi, L. Marconi, C. Silvestre, and R. Cunha, "Robust take-off for a quadrotor vehicle," IEEE Trans. on Robotics, vol. 28, no. 3, pp. 734-742, 2012.

[2] D. Mellinger, N. Michael, and V. Kumar, "Trajectory generation and control for precise aggressive maneuvers with quadrotors," The Int. Journal of Robotics Research, vol. 31, no. 5, pp. 664-674, 2012.

[3] M. W. Mueller, M. Hehn, and R. D'Andrea, "A computationally efficient algorithm for state-to-state quadrocopter trajectory generation and feasibility verification," in 2013 IEEE/RSJ Int. Conf. on Intelligent Robots and Systems, Tokyo, Japan, Nov. 2013, pp. 3480-3486.

[4] A. different look at output tracking: control of a VTOL aircraft, "P. martin and s. devasia and b. paden," Automatica, vol. 32, no. 1, pp. 101-107, 1996.

[5] S. Lupashin and R. D'Andrea, "Stabilization of a flying vehicle on a taut tether using inertial sensing," in 2013 IEEE/RSJ Int. Conf. on Intell. Robots and Systems, Tokyo, Japan, Nov 2013, pp. 2432-2438.

[6] M. Tognon and A. Franchi, "Nonlinear observer-based tracking control of link stress and elevation for a tethered aerial robot using inertialonly measurements," in 2015 IEEE Int. Conf. on Robotics and Automation, Seattle, WA, May 2015, pp. 3994-3999.

[7] M. Tognon, S. S. Dash, and A. Franchi, "Observer-based control of position and tension for an aerial robot tethered to a moving platform,' IEEE Robotics and Autom. Letters, vol. 1, no. 2, pp. 732-737, 2016.

[8] S.-R. Oh, K. Pathak, S. K. Agrawal, H. R. Pota, and M. Garrett, "Autonomous helicopter landing on a moving platform using a tether," in 2005 IEEE Int. Conf. on Robotics and Automation, Barcelona, Spain, Apr. 2005, pp. 3960-3965.

[9] L. Sandino, D. Santamaria, M. Bejar, A. Viguria, K. Kondak, and A. Ollero, "Tether-guided landing of unmanned helicopters without GPS sensors," in 2014 IEEE Int. Conf. on Robotics and Automation, Hong Kong, China, May 2014, pp. 3096-3101.

[10] M. Tognon, A. Testa, E. Rossi, and A. Franchi, "Extended experiments for robust takeoff and landing on slopes exploiting a passive tether," LAAS-CNRS, Tech. Rep. hal-01349743, July 2016.

[11] A. Censi, A. Franchi, L. Marchionni, and G. Oriolo, "Simultaneous maximum-likelihood calibration of odometry and sensor parameters," IEEE Trans. on Robotics, vol. 29, no. 2, pp. 475-492, 2013.

[12] M. Tognon and A. Franchi, "Nonlinear observer for the control of bitethered multi aerial robots," in 2015 IEEE/RSJ Int. Conf. on Intelligent Robots and Systems, Hamburg, Germany, Sep. 2015, pp. 1852-1857. 